ARTICLE

\title{
Transformation of hard pollen into soft matter
}

\author{
Teng-Fei Fan ${ }^{1,4}$, Soohyun Park (10 1,4, Qian Shi ${ }^{2}$, Xingyu Zhang ${ }^{3}$, Qimin Liu ${ }^{3}$, Yoohyun Song ${ }^{1}$, Hokyun Chin ${ }^{1}$, \\ Mohammed Shahrudin Bin Ibrahim¹, Natalia Mokrzecka1, Yun Yang ${ }^{1}$, Hua Li ${ }^{3 凶}$, Juha Song (1) ${ }^{2 凶}$, \\ Subra Suresh ${ }^{1 凶} \&$ Nam-Joon Cho (i) ${ }^{1,2 \otimes}$
}

Pollen's practically-indestructible shell structure has long inspired the biomimetic design of organic materials. However, there is limited understanding of how the mechanical, chemical, and adhesion properties of pollen are biologically controlled and whether strategies can be devised to manipulate pollen beyond natural performance limits. Here, we report a facile approach to transform pollen grains into soft microgel by remodeling pollen shells. Marked alterations to the pollen substructures led to environmental stimuli responsiveness, which reveal how the interplay of substructure-specific material properties dictates microgel swelling behavior. Our investigation of pollen grains from across the plant kingdom further showed that microgel formation occurs with tested pollen species from eudicot plants. Collectively, our experimental and computational results offer fundamental insights into how tuning pollen structure can cause dramatic alterations to material properties, and inspire future investigation into understanding how the material science of pollen might influence plant reproductive success.

\footnotetext{
${ }^{1}$ School of Materials Science and Engineering, Nanyang Technological University, 50 Nanyang Avenue, Singapore 639798, Singapore. ${ }^{2}$ School of Chemical and Biomedical Engineering, Nanyang Technological University, 62 Nanyang Drive, Singapore 637459, Singapore. ${ }^{3}$ School of Mechanical and Aerospace Engineering, Nanyang Technological University, 50 Nanyang Avenue, Singapore 639798, Singapore. ${ }^{4}$ These authors contributed equally: Teng-Fei Fan,

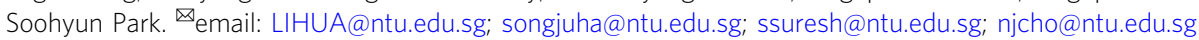


$\mathrm{P}$ ollen is a remarkable natural material that plays a critical role in plant reproduction and transfers viable cellular material (i.e., male gametes or sperm cells) between different reproductive parts of plants ${ }^{1,2}$. Regarded as practically indestructible 3,4 , pollen grains are highly dynamic microscale structures that possess unique material characteristics ${ }^{5-7}$. When pollen grains are released from anthers, they become dehydrated and individual grains fold onto themselves-a structurally intricate process termed "harmomegathy" 8,9 . In addition, pollen grains undergo architectural remodeling during pollen tube growth, which is guided by an organized sequence of enzymatically controlled reactions ${ }^{10,11}$. Expanding upon the biochemical mechanisms that plants use to control the chemorheological properties of pollen grains holds untapped potential for engineering polleninspired materials with high-performance capabilities.
Common features of pollen grains across various plant species include a microcapsule structure, function-driven shape, and ornamental architecture ${ }^{12}$. We selected pollen grains from sunflower plants (Helianthus annuus), which have spiky appendages and a tripartite structure (Fig. 1a and Supplementary Fig. 1). The outermost layer ("exine") is made up of sporopollenin, which is a strong, crosslinked biopolymer", while the inner layer ("intine") is composed of elastic, load-bearing cellulose/hemicellulose microfibrils and pectin ${ }^{13,14}$ (Fig. 1b). The aperture gap in the exine layer is integral to pollen tube growth and is neighbored by pollen wall regions with distinct material properties. As part of remodeling processes, pectin methylesterase (PME) enzyme plays a key role in controlling wall elasticity by converting pectin into pectate $^{15}$, exposing carboxylic acid functional groups and imparting greater surface charge density that modulates the intine structural
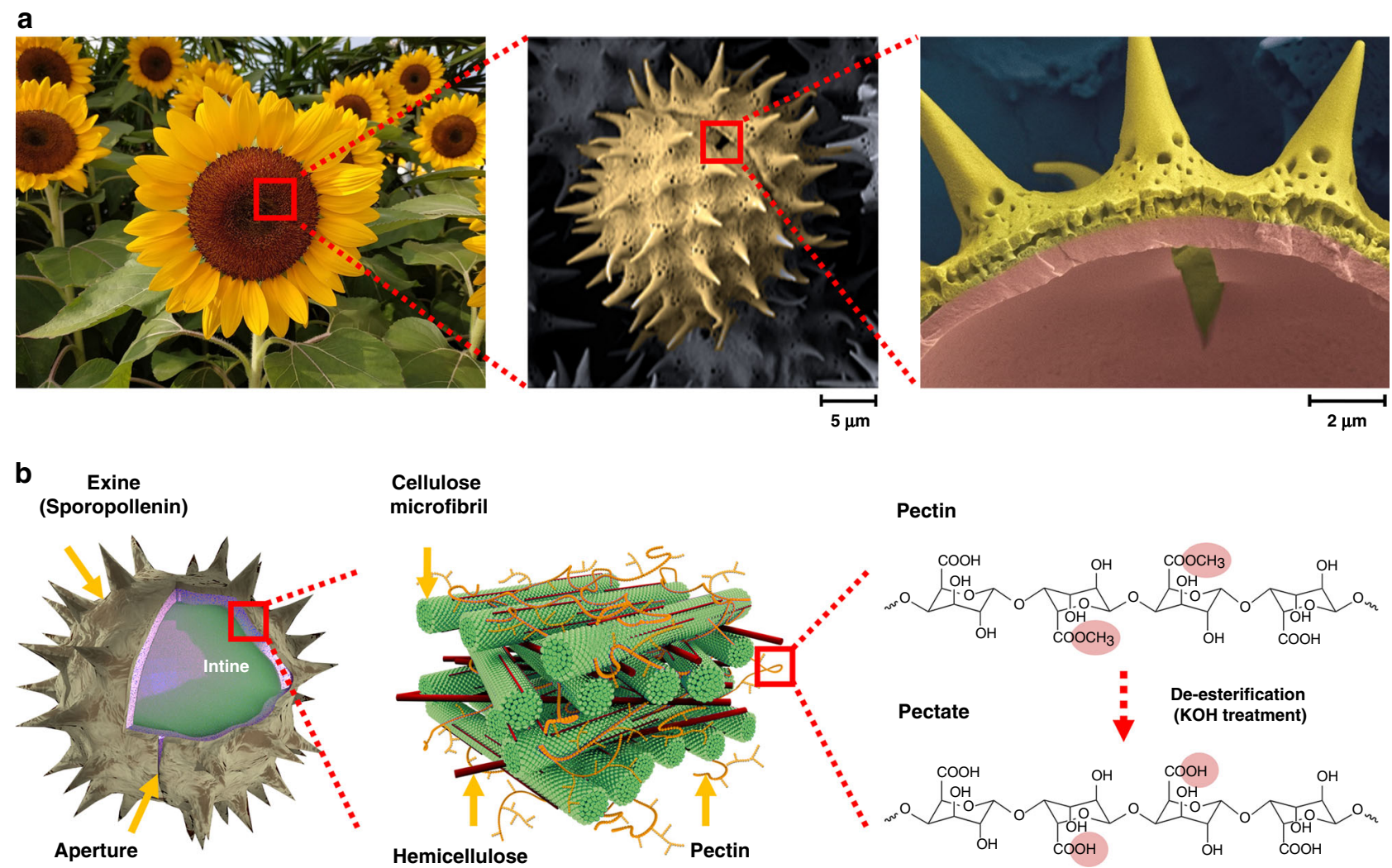

Cellulose

microfibril

Pectin
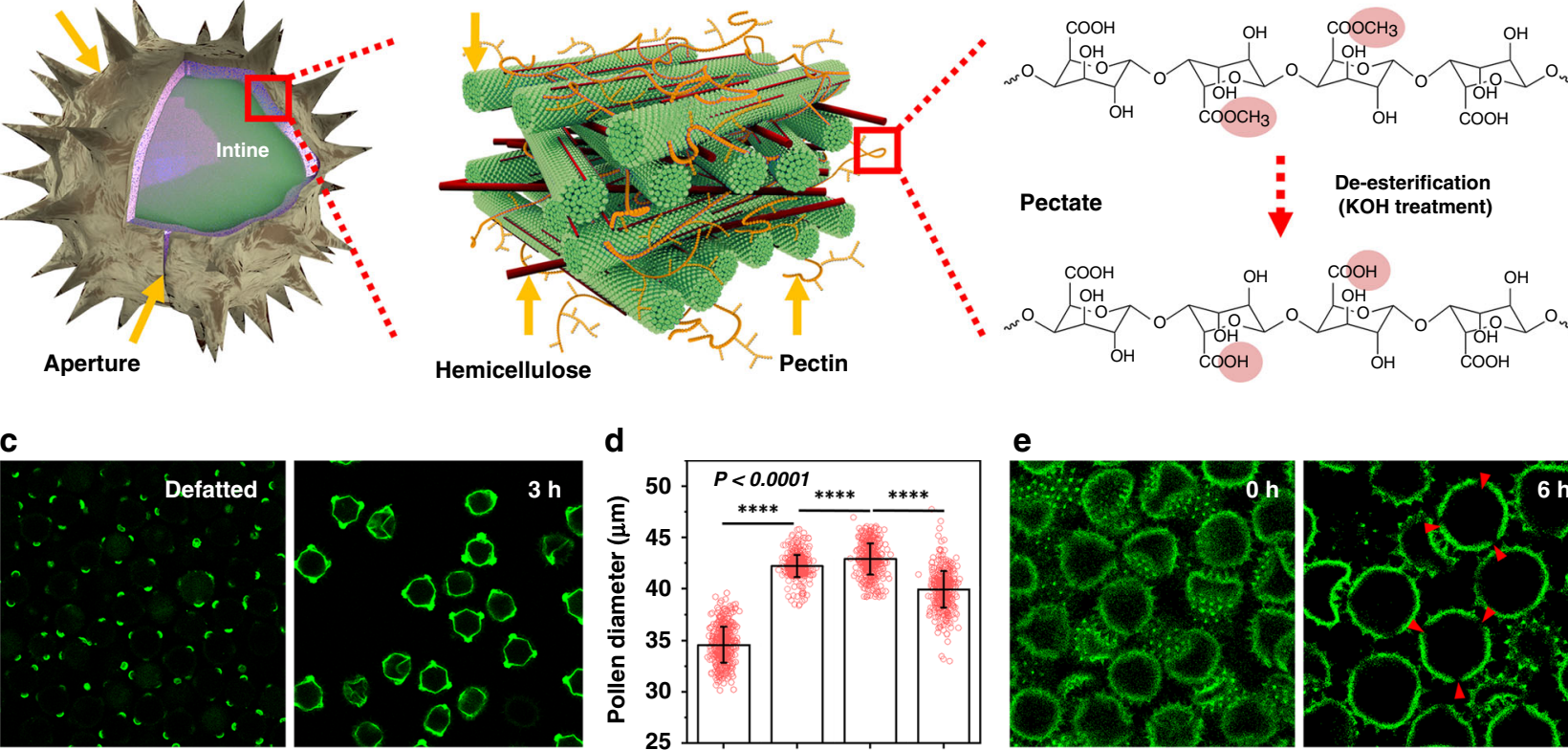

\section{d}
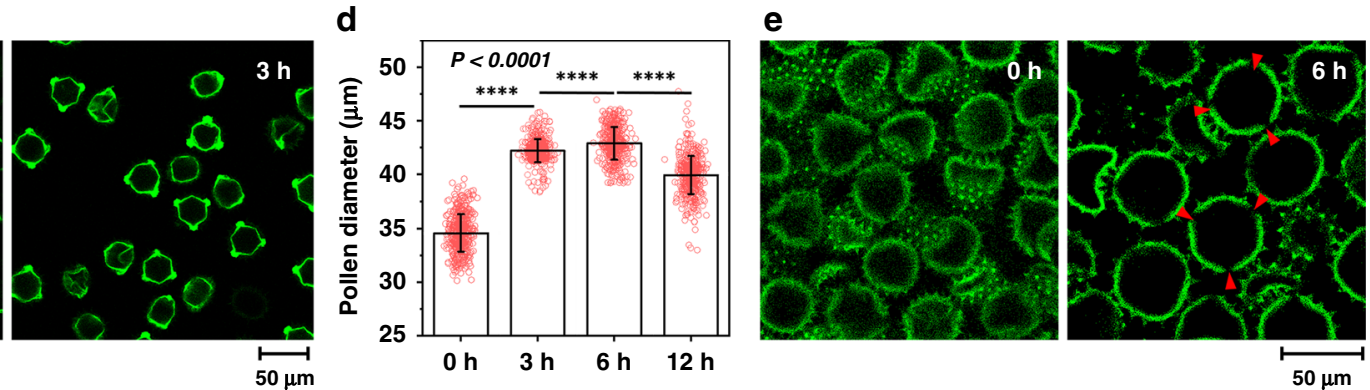

Fig. 1 Engineering dynamic responsiveness in pollen particles. a Steps involved in the extraction of pollen grains from sunflower plants, shown here with cross-sectional SEM images. The pollen structures are pseudo-colored. b Schematic illustration of stimuli-responsive pollen capsule behavior at multiple length scales. Left: Pollen structural components. Middle: Cellulose microfibrils organized by hemicellulose and pectin in the intine layer. Right: Hydrolytic conversion of pectin to pectate within the intine layer. c Immunofluorescence microscopy detection of de-esterified pectin within pollen shells using JIM5 as the primary antibody. d Size characterization of treated pollen particles as a function of KOH incubation time, as measured by DIPA. Data are analyzed by Student's $t$-test and are presented as mean \pm s.d. $\left(n=500\right.$ per condition). ${ }^{\star \star \star \star} p<0.0001$. Source data are provided as a Source Data file. e Crosssectional CLSM images of pollen-derived microgel particles. Red arrows indicate aperture openings. 
arrangement ${ }^{15}$. Biologically, this enzymatic activity is spatially controlled within the pollen wall structure ${ }^{16,17}$, and inspires biomimetic strategies to engineer pollen structures with tunable material properties.

Motivated by these intricate biological features, we developed a nature-inspired strategy to de-esterify pectin molecules throughout the entire pollen wall structure. Our strategy involved two stages focused on liberating pollen epitopes, followed by chemical hydrolysis. In order to expose pectin epitopes, the defatted pollen was first treated with $10 \%$ (wt/vol) $\mathrm{KOH}$ at $80^{\circ} \mathrm{C}$ for $2 \mathrm{~h}$ under stirring to efficiently remove cytoplasmic components. Afterwards, the pollen was incubated without stirring in fresh $10 \%$ (wt/vol) $\mathrm{KOH}$ at $80^{\circ} \mathrm{C}$ for an extended period of up to $12 \mathrm{~h}$ before centrifugation was performed and this combination of processing steps facilitated microgel formation (Supplementary Fig. 2).

\section{Results}

Characterization of de-esterified pollen particles. To verify deesterification, we conducted immunofluorescence microscopy experiments using the monoclonal JIM5 antibody that recognizes weakly esterified epitopes of pectin ${ }^{18}$ (Fig. 1c). The results confirmed successful processing as indicated by increased deesterification after $3 \mathrm{~h}$ incubation while longer incubation periods resulted in more extensive de-esterification of pectin molecules ${ }^{19}$ (Supplementary Fig. 3a). Dynamic image particle analysis (DIPA) further revealed that the treated particles swelled from approximately 35 to $43 \mu \mathrm{m}$ in diameter after $6 \mathrm{~h}$ incubation, which was judged to be the optimal condition based on the greatest extent of particle swelling (Fig. 1d). These results were complemented by immunolabeling experiments with JIM7 antibody, which recognizes highly esterified epitopes of pectin ${ }^{18}$ (Supplementary Fig. 3b). In addition, the pollen dispersions exhibited more gel-like character, as indicated by increased resistance to gravity due to de-esterification of pectin (Supplementary Fig. 3c). Coinciding with an increase in gel-like properties, confocal laser scanning microscopy (CLSM) experiments revealed that chemical processing caused the pollen particles to swell and join together while individual particles remained structurally intact ${ }^{20}$ (Fig. 1e). Fourier transform infrared (FTIR) spectroscopy experiments also verified that all $\mathrm{KOH}$-treated pollen grains appear nearly identical, irrespectively of the treatment time, whereas only the defatted sample exhibited more complex and convoluted spectral features along with peaks corresponding to pectin molecules due to residual cytoplasmic contents (Supplementary Fig. 4). Taken together, the findings reveal that incubating pollen in well-controlled alkaline conditions for extended periods of time (replicating classical soapmaking ${ }^{21}$ ) can result in the transformation of hard pollen grains into pliable, soft microgel particles.

Chemomechanical responsiveness of pollen microgel particles. To further evaluate how de-esterification affects the chemomechanical responsiveness of individual pollen particles, we developed a tethering strategy to immobilize pollen particles on a glass surface. While the carboxylic acid functional groups on the de-esterified pectin molecules are expected to be protonated under acidic $\mathrm{pH}$ conditions, the same functional groups would exhibit a higher degree of deprotonation, and hence greater anionic surface charge, under increasingly basic $\mathrm{pH}$ conditions (Fig. 2a). Thus, we exposed the immobilized particles to rapid changes in solution $\mathrm{pH}$ and observed morphological changes by time-lapse optical microscopy (Fig. 2b). When the solution $\mathrm{pH}$ was changed from 2 to 12 , the microgel particles underwent extensive swelling and this behavior was reversible when the $\mathrm{pH}$ titration was conducted in the opposite direction (Supplementary Figs. 5-7 and Supplementary Movie 1). The apertures also became swollen (Supplementary Fig. 8). In marked contrast, when the solution $\mathrm{pH}$ was changed from 12 to 2 , the microgel particles underwent relatively faster de-swelling or compression while defatted, unprocessed pollen grains exhibited negligible $\mathrm{pH}$ responsive behavior in both directions (Fig. 2c). The $\mathrm{pH}-$ dependent swelling response is analogous to that of ionizable polymer networks whose time scales for de-swelling occur similarly within seconds 22,23 . The observed behavior also appears to be distinct from harmomegathy because it is triggered by a different stimulus and involves more uniform morphological responses. DIPA experiments confirmed similar $\mathrm{pH}$-dependent effects for pollen microgel particles in bulk solution (Supplementary Fig. 9).

We also evaluated the effect of different ions in altering the swelling/de-swelling properties of pollen microgel particles. Under neutral $\mathrm{pH}$ conditions, the carboxylic acid functional groups in the pectin molecules are predominately deprotonated $^{24}$ and thus sensitive to divalent cations ${ }^{25}$ (Fig. $2 \mathrm{~d}$ ). Timelapse optical microscopy experiments on immobilized pollen particles provided direct evidence of the dynamic response to calcium ions (Fig. 2e). Similar effects were observed with other cations as well (Fig. 2f and Supplementary Movies 2-6). In all cases, the addition of $10 \mathrm{mM}$ ethylenediaminetetraacetic acid (EDTA) - a known cation chelator-caused re-swelling of the microgel particles.

We also investigated how microgel formation affects the structural and mechanical properties of the pollen substructure layers. Scanning electron microscopy (SEM) imaging confirmed the gradual removal of pollen cement from the exine layer-a process that also occurs upon the natural hydration of pollen during germination-with increasing $\mathrm{KOH}$ incubation time ${ }^{26}$ (Supplementary Fig. 10). Moreover, atomic force microscopy (AFM) measurements revealed that prolonged alkaline treatment caused a significant decrease in the Young's modulus of the exine layer (Supplementary Figs. 11 and 12). In turn, the ratio of the Young's modulus values of the exine and intine layers $\left(M_{E / I}\right)$ decreased from $\sim 3$ (defatted pollen) to $\sim 1.5$ (12 h KOH-treated pollen).

Deformation mechanism of bi-layered pollen microparticles. To further evaluate how changes in the chemomechanical properties of pollen substructure layers affect the swelling/de-swelling behavior of pollen microgel particles, we also conducted computational simulations based on a multiphysics model that incorporated finite element analysis (FEA $)^{27-29}$. Specifically, the model simulated the extent of pollen intine swelling in different ionic solutions in line with the aforementioned experiments and accounted for the effects of chemo-electro-mechanical coupled fields on inflation/deflation of the exine layer (Supplementary Fig. 13). The pollen intine swelling pressure $(P i$,swelling $)$ was analytically calculated as a function of changes in environmental conditions (i.e., ion types and concentrations) and the computed values used as boundary conditions for analyzing inflation of the exine layer (i.e., exine inflation pressure, $P_{e \text {,inflation }}$ ) to evaluate the swelling/deswelling behavior of pollen microgels, and we systematically studied $M_{E / I}$ ratios from 0.15 to 8 (Supplementary Table 1). A stiffer exine $\left(M_{E / I}>2\right)$ would be expected to impose a rigid boundary condition upon the hydrated intine, thereby reducing the potential for intine swelling (Fig. 3a). On the other hand, when $M_{E / I}<2$, both experiments and simulations demonstrated a steep increase in particle diameter $\left(M_{E / I}=1.6\right.$, Supplementary Movie 7). Moreover, the de-swelling of pollen microgel particles that was experimentally observed in the presence of multivalent cations ( $c f$. Fig. 2) was also captured in the simulations for $M_{E / I}<1$, and arises from the intine becoming stiffer than the exine due to the chelation reaction as shown in Fig. $2 \mathrm{~d}$ 
a
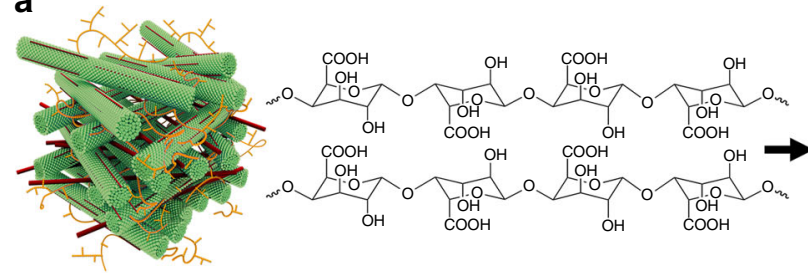
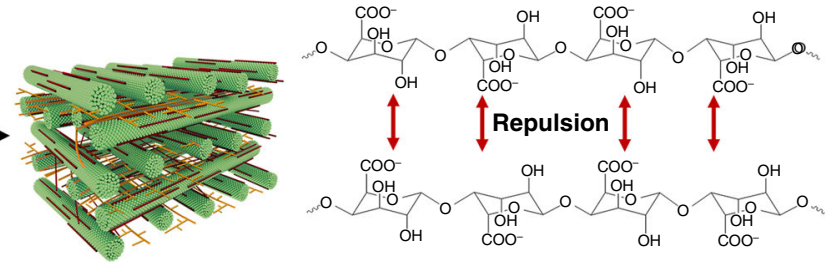

b
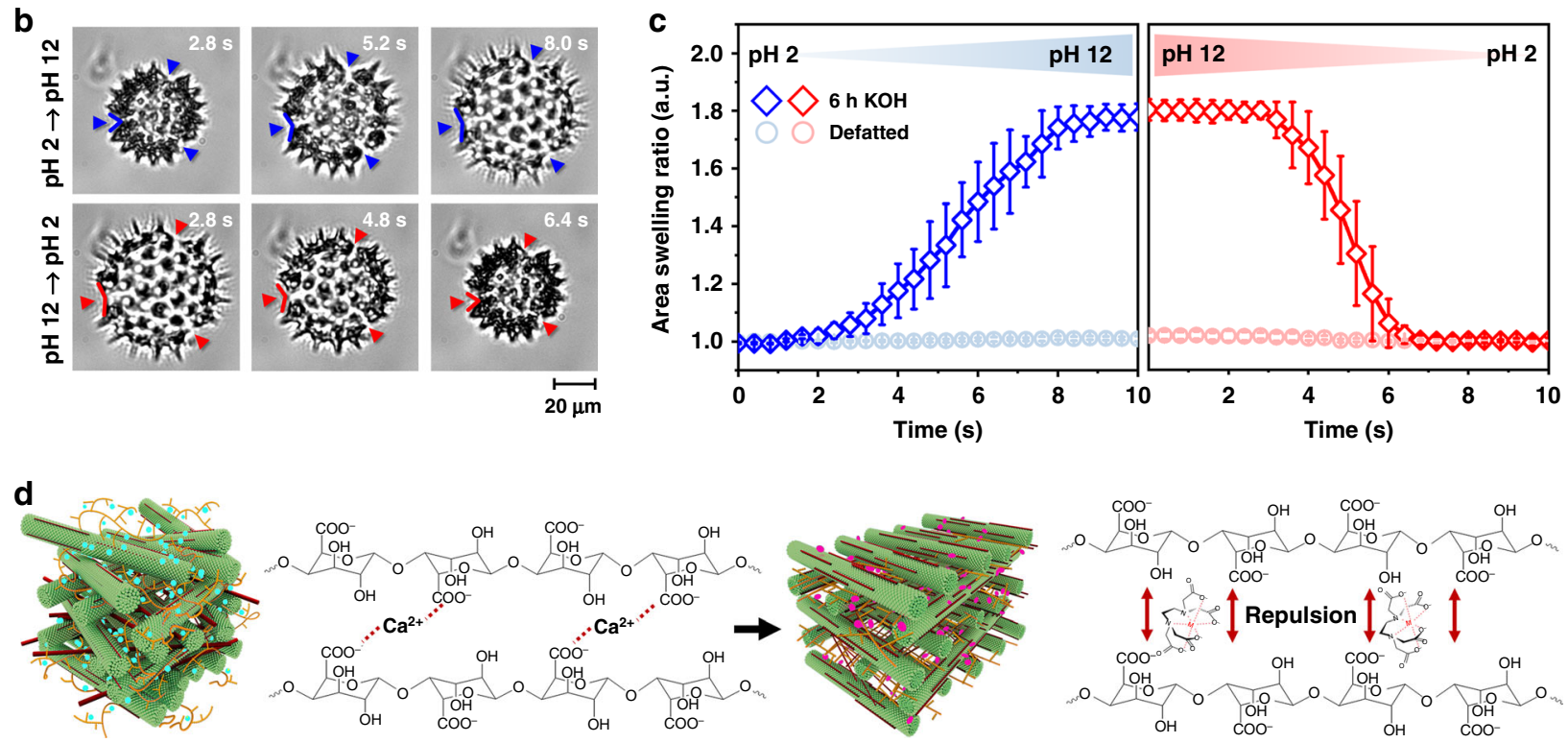

e
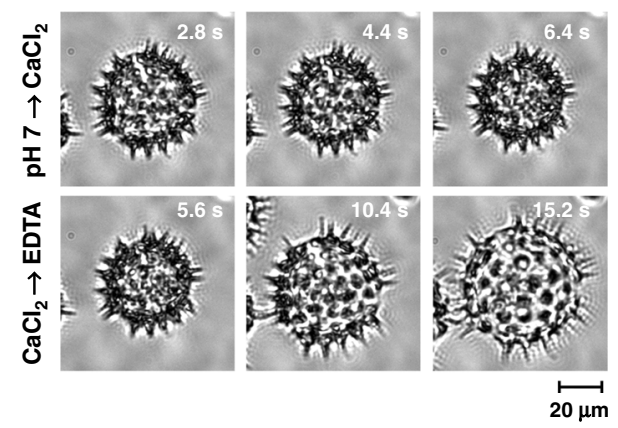

$\mathbf{f}$

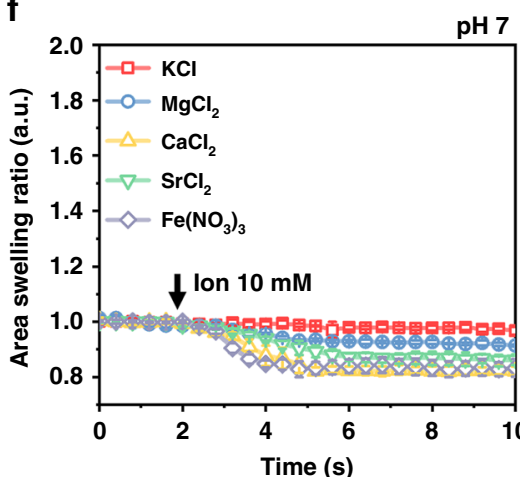

$\mathrm{pH} 7$

Ion $10 \mathrm{mM}$

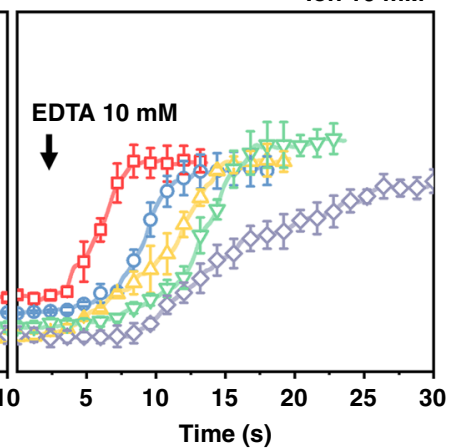

Fig. 2 Tunable pectate interactions enable rapid, stimuli-responsive material properties. a Schematic illustration of $\mathrm{pH}$-dependent effects on pectin structure and corresponding intermolecular repulsion events. b Time-lapse optical micrographs of tethered pollen particles. The solution $\mathrm{pH}$ was changed from $\mathrm{pH} 2$ to 12 (top series), and from pH 12 to 2 (bottom series). c Quantitative comparison of pH-induced pollen swelling and de-swelling behavior. d Schematic illustration of cation-induced attraction and EDTA chelating agent-induced repulsion between pectin molecules. e Time-lapse optical micrographs of tethered pollen particles. Calcium ions were added (top), followed by EDTA chelating agent (bottom). $\mathbf{f}$ Quantitative comparison of ioninduced pollen de-swelling and swelling behaviors. Mean \pm s.d. are reported ( $n=5$ particles) in $\mathbf{c}$ and $\mathbf{f}$, and the area swelling ratio was normalized by the initial area at $\mathrm{pH} 2$ and $\mathrm{pH} 7$ in $\mathbf{c}$ and $\mathbf{f}$, respectively. Source data are provided as a Source Data file.

$\left(M_{E / I}=0.7\right.$, Supplementary Movie 8$)$. Together with the gelation of de-esterified pectin molecules within the intine layer, these findings reveal that a softened exine layer plays an important role in modulating the mechanical properties of microgel particles by allowing greater swelling of the hydrated intine. Thus, the interplay of mechanical responses in the exine and intine layers dictates the morphological behavior of the microgel particles.

Taking into account the mechanical behavior of an individual microgel particle, its representative volume element (RVE) in the FEA was defined as a structure that consisted of a hyperelastic exine layer only with one aperture and one-third symmetry (Supplementary Fig. 13). We captured the large elastic deformation and strain energy density of a microgel particle by using FEA to simulate pollen exine inflation. Numerical simulations also revealed the morphological evolution of a microgel particle during its structural expansion (Fig. 3b, c and Supplementary Figs. 14 and 15). We defined three key swelling ratios of pollen-derived microgel particles: $\lambda_{0}, \lambda_{\text {open }}$, and $\lambda_{\max } . \lambda_{0}$ is the initial swelling ratio $\left(\lambda_{0}=1\right)$ when the total volume of the microgel particle is minimized. Once pectin hydration begins, rapid aperture opening occurs and intine swelling-induced pressure and resulting strain are highly localized at the tips of the three apertures. $\lambda_{\text {open }}$ is the critical swelling ratio of a pollen microgel particle, at which these chemomechanical shifts trigger 

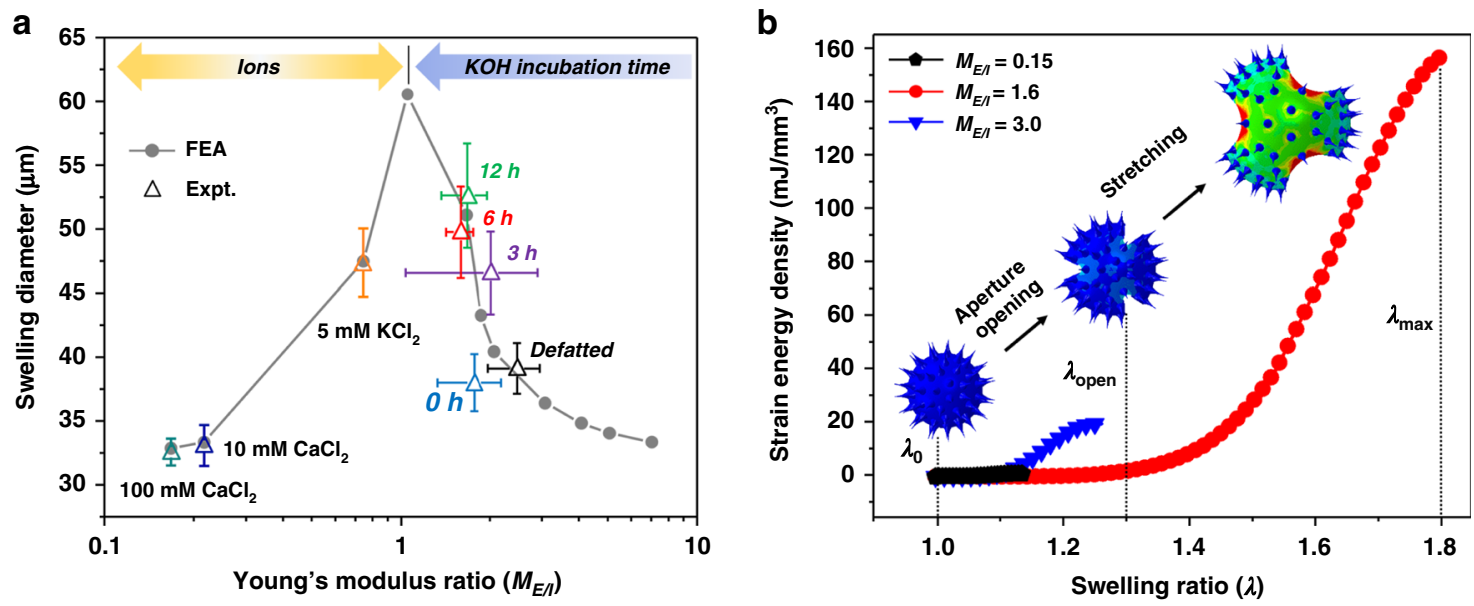

C
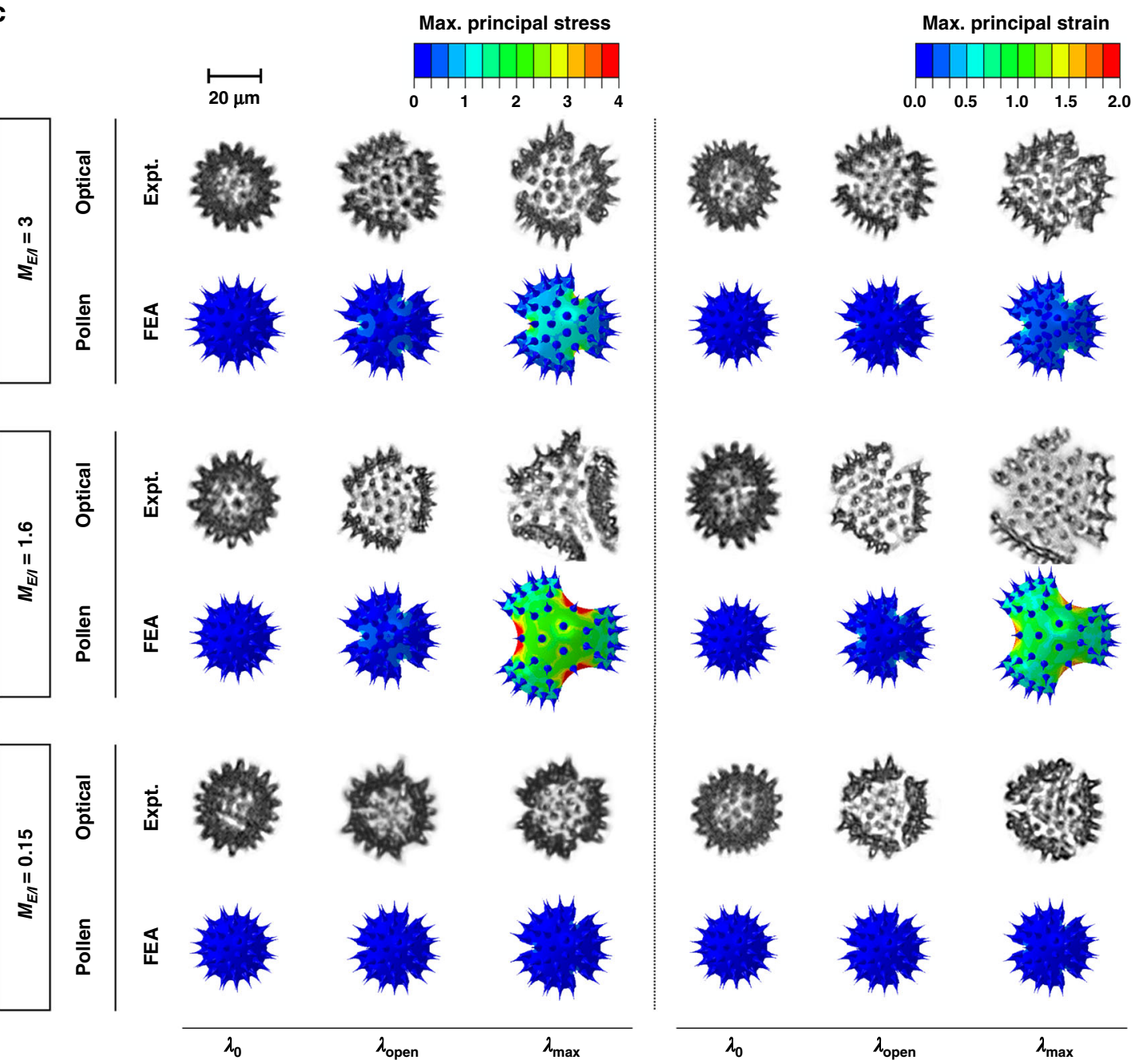

Fig. 3 Mechanical response of pollen microgel particles. a Swelling diameter of pollen microgel particle as a function of the ratio of the Young's modulus values of the exine to intine layers, $M_{E / /}$. b Predicted evolution of the strain energy density during pollen expansion as a function of the swelling ratio ( $\lambda$ ) for three typical Young's modulus ratio values $\left(M_{E / /}=0.15,1.6\right.$, and 3). c Maximum principal stress and maximum principal strain contours of the pollen microgel particles $\left(M_{E / I}=0.15,1.6\right.$, and 3$)$ for three critical swelling ratios $\left(\lambda_{0}, \lambda_{\text {open, }}\right.$ and $\left.\lambda_{\text {max }}\right)$ (labeled FEA), along with representative optical micrographs of pollen microgel particles in various chemical environments (i.e., ionic changes) that triggered similar morphological evolutions. $M_{E / I}=0.15$ corresponds to pollen microgel particles immersed in $100 \mathrm{mM} \mathrm{CaCl} ; M_{E / I}=1.6$ to $6 \mathrm{~h} \mathrm{KOH}$-treated pollen microgels incubated in $\mathrm{pH} 10$ solution; $M_{E / I}=3$ to defatted pollen grains. Source data are provided as a Source Data file. 
opening of the three apertures. When the critical swelling ratio exceeds $\lambda_{\text {open }}$, the deformation mode of a microgel particle transfers from aperture opening to stretching. Further expansion of the microgel particle induces large deformation of both the intine and exine layers until the maximum swelling ratio, $\lambda_{\max }$, is reached. The aperture opening does not require significant strain energy with highly localized deformation around the apertures, whereas subsequent stretching is an energy-consuming process that involves large-scale global deformation throughout the pollen shell. Stress and strain contours of $6 \mathrm{~h} \mathrm{KOH}$-treated pollen microgel particles immersed in $\mathrm{pH} 10$ solution $\left(M_{E / I}=1.6\right)$ indicate that the three apertures are initially opened, accompanied by a small volume change until the critical swelling ratio $\lambda_{\text {open }}$ is reached, followed by a dramatic change in microgel particle diameter at the maximum swelling ratio, $\lambda_{\max }$ (Supplementary Movie 7). In the case of small expansion $\left(M_{E / I}<0.5\right.$ or $M_{E / I}>2$ ), the stress and strain of a microgel particle are localized around the aperture tips, indicating that either a stiffer exine or intine alone does not accommodate stretching of a particle, thereby constraining its expansion and easily recovering its dry condition with minimum energy loss.

Microgel formation among different plant kingdom. To extend our findings, we tested pollen grains and spores from other clades and discovered that those from flowering monocots (cattail) and gymnosperms (pine), as well as spore-bearing lycophytes (lycopodium) did not form microgel particles (Fig. 4). We also tested pollen grains from baccharis and camellia plants, which belong to the same eudicot clade as sunflower plants (Fig. 4a and Supplementary Fig. 16). We discovered that pollen grains from tested eudicot plants are transformed into microgel particles (Fig. 4b, Supplementary Figs. 17-28 and Supplementary Movie 9). Taken together, these results support that pollen grains from the tested eudicot plants have suitable material properties to facilitate microgel conversion using our nature-inspired strategy. The observed variation in gelling propensity among pollen from different plant species may also relate to structural variations in microcapsule architecture that give rise to distinct chemomechanical responses.

Microgel formation among pollen species of eudicot plants lends strong support to the important role of chemomechanical responses in controlling pollen-related biological functions. Indeed, our strategy involving de-esterification of pectin molecules within the intine layer mimics the activity of key enzymes involved in pollen tube growth, and our findings demonstrate how subtle, chemically programmed variations in the mechanical properties of both the exine and intine layers can cause the swelling of pollen particles akin to the shape transformations that occur during the orchestrated sequence of pollen hydration, germination, and tube growth ${ }^{30}$. While experimental and computational studies of dehydration-induced harmomegathy have long recognized that the exine and intine layers of natural pollen grains have distinct mechanical properties ${ }^{8}$, we discovered that this distinction alone is insufficient to explain hydrationinduced pollen swelling. Strikingly, through direct experimental investigation with SEM and AFM and computational models that verified changes in the chemomechanical properties of pollen substructure layers drive the stimuli-responsive behavior of microgel particles, our findings reveal that the interplay of exine and intine layers plays a key role in dictating pollen swelling. The ratio of the Young's modulus values of the chemically tuned exine and intine layers must be within a certain, optimal range to trigger significant swelling and volume expansion of pollenderived microgel particles in our experiments. This chemomechanical balance appears to be carefully regulated and suggests

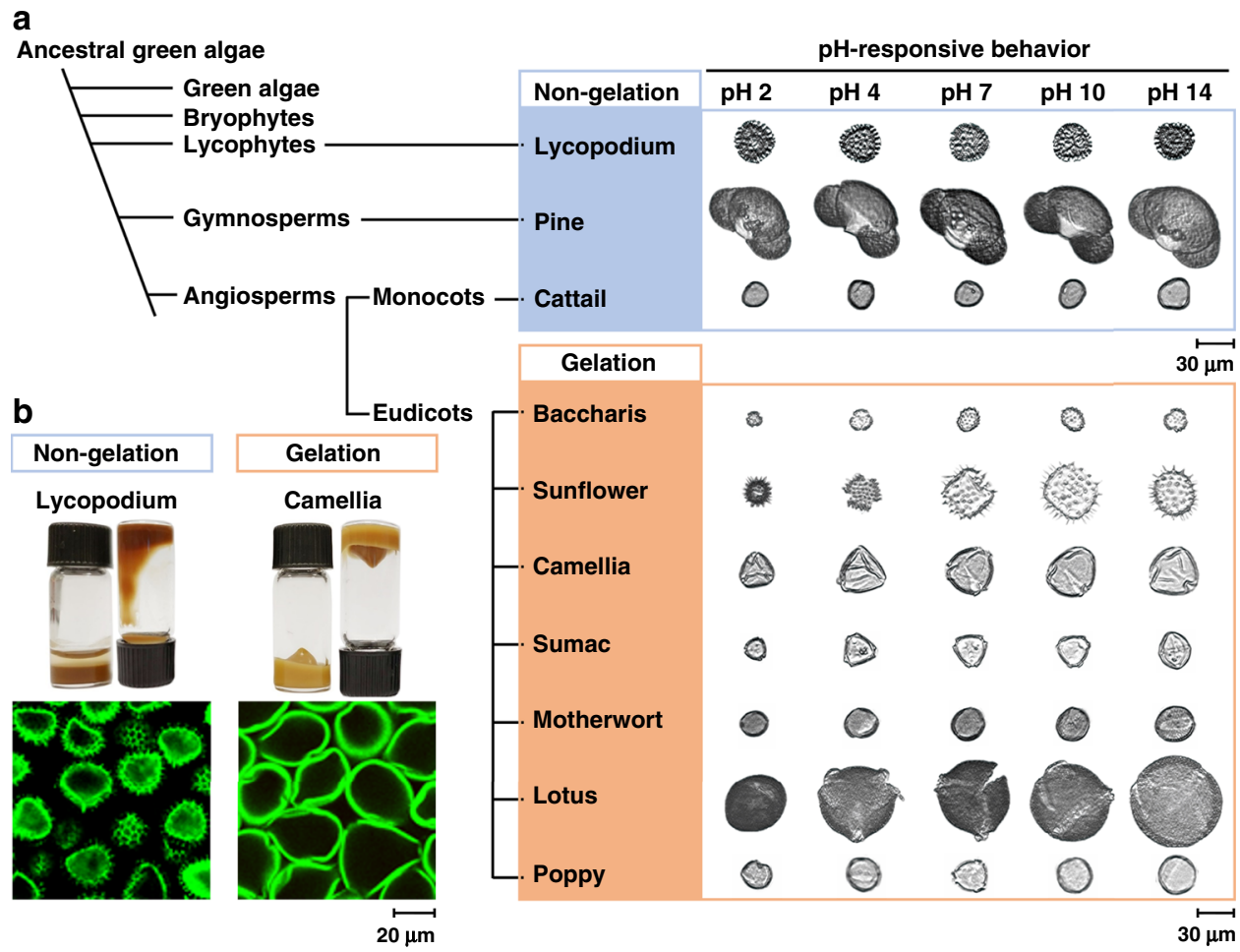

Fig. 4 Plant kingdom sampling to identify other types of chemically tractable pollen. a Evolutionary cladogram of plant pollen sources including lycophytes, gymnosperms and angiosperms with corresponding DIPA images as a function of pH response. $\mathbf{b}$ Top: Photographs of pollen dispersion in upright and reversed vials, showing the response of pollen gels to gravity. Bottom: Cross-sectional CLSM images of treated particles in cases where particles interact to form a microgel and where particles remain as discrete, individual entities. 


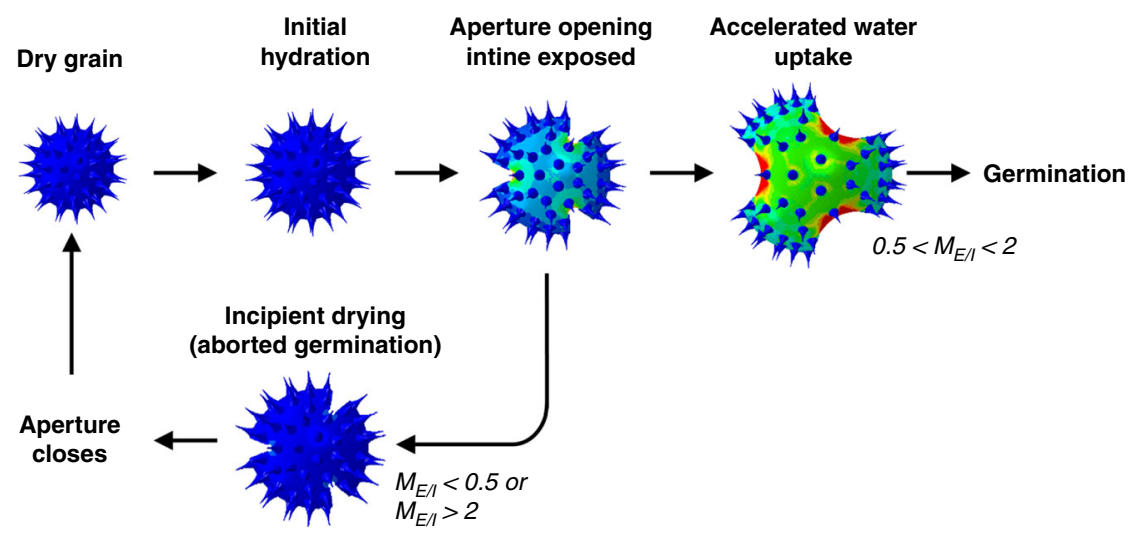

Fig. 5 Pollen hydration/dehydration cycle of angiosperms with corresponding FEA simulation images. The top sequence shows a dry pollen grain undergoing the water uptake process during germination. In situations where complete hydration is not possible, germination might be aborted, and the apertures close again as shown in the bottom sequence ${ }^{13}$.

there is a chemorheological regulatory pathway at the individual particle level underpinning whether pollen hydration leads to successful germination or incipient drying ${ }^{10}$ (Fig. 5).

\section{Discussion}

In addition to the foregoing mechanistic implications, our results also have potential significance for pollen-inspired design of materials for a variety of possible applications. For example, hard pollen particles have been explored as natural hollow microcapsules owing to a number of factors including their abundant and renewable supply, low cost, robust shell structure, chemical stability, biocompatibility, high monodispersity, quality control, and species-specific particle size and ornamental features ${ }^{31,32}$. The present findings broadly extend this potential by demonstrating how a simple chemical process akin to classical soapmaking can convert ultratough pollen grains into stimuliresponsive microgel particles.

Understanding and tuning the biomechanics of natural materials holds promise for materials design and application. These findings are relevant to understanding how the morphological evolution of microgel particles might play a role in ensuring plant reproduction in nature ${ }^{10}$. The ease of aperture opening in pollen particles induces rapid water uptake from the stigma surface. Meanwhile, the subsequent stretching and expansion of pollen grains require significant water uptake and considerable structural change with high energy consumption, to promote germination. As a result, insufficient hydration of pollen due to the inappropriate stigma conditions ceases germination, leading to harmomegathy with minimum energy loss (Fig. 5). Thus, our findings provide insight into the design principles of pollen grains in the context of harmomegathy (dehydration) and germination (hydration), while explaining how our microgel formation and tuning approach can exceed the performance limits of these natural processes. Furthermore, the process offers new pathways to produce highly uniform microgel particles from pollen sources. Such particles could be useful in a wide range of applications where excellent quality control is essential as, for example, in high-performance sensors and actuators, as well as in vivo drug delivery.

\footnotetext{
Methods

Pollen materials. Defatted pollen grains from the sunflower (Helianthus annuus L.), pine (Pinus taeda), and daisy (Baccharis halimifolia L.) families were purchased from Greer Laboratories, Inc. (Lenoir, NC, USA). Natural lycopodium (Lycopodium clavatum) spores (S-type) and the cattail (Typhae angustfolia) pollen grains were purchased from Sigma-Aldrich (St. Louis, MO, USA), and camellia (Camellia Sinensis L.) bee pollen granules were purchased from Yuensun Biological
}

Technology Co., Ltd. (Xi'an, Shaanxi, China). Sumac (Rhus chinensis) bee pollen was purchased from Fengzhixiang Apiculture Co. Ltd. (Zhengzhou, Henan, China) Motherwort (Leonurus cardiaca) bee pollen was purchased from GTL Biotech Co., Ltd. (Xi'an, Shaanxi, China). Lotus (Nelumbo nucifera) bee pollen and poppy (Papaver rhoeas) pollen were purchased from Peffer Industrial Co., Ltd. (Zhengzhou, Henan, China).

Microgel preparation. Pollen microgel suspensions were prepared following a three-step process:

(1) Defatting: Natural pollen grains or bee pollen granules were defatted to remove pollenkitt. For this purpose, bee pollen granules $(250 \mathrm{~g})$ were refluxed in acetone $(500 \mathrm{~mL})$ for $3 \mathrm{~h}$ in a round-bottom flask under magnetic stirring $\left(50{ }^{\circ} \mathrm{C}, 220 \mathrm{rpm}\right)$. Then, acetone was decanted and deionized water $(1 \mathrm{~L}$, $50^{\circ} \mathrm{C}$ ) was added to the sample, mixed, and stirred for $1 \mathrm{~h}$. The pollen suspension was passed through a nylon mesh (with $300 \mu \mathrm{m}$ diameter pores) to remove any contaminating particulate matter. The resulting filtrate was subsequently passed through filter paper (with $6 \mu \mathrm{m}$ diameter pores) in a vacuum filtration system. Next, the pollen sample was hydrated with deionized water $\left(1 \mathrm{~L}, 50^{\circ} \mathrm{C}\right)$ with magnetic stirring for $30 \mathrm{~min}$ and then vacuum filtered. The resulting pollen particles were refluxed in acetone $\left(500 \mathrm{~mL}, 50^{\circ} \mathrm{C}\right)$ with magnetic stirring $(400 \mathrm{rpm})$ for $3 \mathrm{~h}$. Afterward, acetone was removed by vacuum filtration and pollen samples were transferred to a glass petri dish and left to dry in a fume hood $(12 \mathrm{~h})$. After defatting with acetone, a dried pollen sample $(20 \mathrm{~g})$ was dispersed in diethyl ether $(250 \mathrm{~mL})$ with magnetic stirring $\left(25^{\circ} \mathrm{C}, 400 \mathrm{rpm}, 2 \mathrm{~h}\right)$ in order to defat the samples. This diethyl ether defatting step was done twice and fresh diethyl ether was used in each cycle. Removal of diethyl ether was done by vacuum filtration. After washing with diethyl ether twice, the pollen sample was dispersed in fresh diethyl ether and left to stir overnight $\left(25^{\circ} \mathrm{C}, 400 \mathrm{rpm}, 12 \mathrm{~h}\right)$. Diethyl ether was then removed by vacuum filtration and the pollen sample was transferred to a petri dish and air-dried in a fume hood $(12 \mathrm{~h})$.

(2) Cytoplasmic removal (1st $\mathrm{KOH}$ treatment step): Defatted pollen (2 g) was mixed with aqueous $10 \%$ (wt/vol) $\mathrm{KOH}(20 \mathrm{~mL}$ ) in a $100 \mathrm{~mL}$ polytetrafluoroethylene (PTFE) round-bottom flask under magnetic stirring at $400 \mathrm{rpm}$. The suspension was refluxed for $2 \mathrm{~h}$ at $80^{\circ} \mathrm{C}$ with stirring at 200 $\mathrm{rpm}$. The suspension was transferred to a $50 \mathrm{~mL}$ conical centrifuge tube, and then centrifuged at $5000 \times g$ for $5 \mathrm{~min}$. The supernatant was removed and the sample was topped up to a total volume of $40 \mathrm{~mL}$ with fresh $10 \%$ (wt/vol) $\mathrm{KOH}$. The mixture was vortexed at high speed for $2 \mathrm{~min}$, followed by centrifugation at $5000 \times g$ for $5 \mathrm{~min}$. The $\mathrm{KOH}$ washing step was repeated a total of 5 times. Finally, the supernatant was decanted and the pollen suspension was left in the tube for the next treatment step.

(3) Microgel formation (2nd $\mathrm{KOH}$ treatment step): Fresh $10 \%$ (wt/vol) $\mathrm{KOH}$ was added to the pollen sample up to a total volume of $40 \mathrm{~mL}$, followed by vortexing at high speed for $2 \mathrm{~min}$. The sample was left to sit in a hot plate oven set to $80^{\circ} \mathrm{C}$ for a specific period of time $(3,6$, or $12 \mathrm{~h})$. After that, the pollen particles were separated by centrifugation $(5000 \times g, 5 \mathrm{~min})$. The supernatant was removed and the $50 \mathrm{~mL}$ conical centrifuge tube was topped up with deionized water (up to a total volume of $40 \mathrm{~mL}$ ), followed by vortexing at high speed $(2500 \mathrm{rpm})$ for $2 \mathrm{~min}$. The resulting pollen suspension was centrifuged again $(5000 \times g, 5 \mathrm{~min})$ and the supernatant was removed to measure its $\mathrm{pH}$ level using $\mathrm{pH}$-indicator strips (MilliporeSigma, Burlington, MA, USA). These water-washing steps were repeated until the $\mathrm{pH}$ level reached 7.5 . The resulting pollen microgel suspension was collected by the last step of centrifugation followed by the supernatant removal, then stored at $4^{\circ} \mathrm{C}$ for further characterization. While 
alkaline processing conditions can be used to convert hard pollen grains into soft microgel particles, not all alkaline processing protocols work and different results such as particle fragmentation (see, e.g., ref. ${ }^{33}$ ) can occur depending on processing parameters such as incubation time, static or stirred incubation conditions, washing solvents, and drying procedures.

Dynamic image particle analysis. Dynamic image particle analysis was performed using a benchtop Fluid Imaging FlowCAM system (Fluid Imaging Technologies, Scarborough, ME, USA) with a $200 \mu \mathrm{m}$ flow cell and a $\times 10$ optical lens. To optimize the focus and other measurement settings, the system was calibrated with $50 \mu \mathrm{m}$ diameter polystyrene beads (Thermo Fisher Scientific, Waltham, MA, USA). The liquid sample was injected into the flow chamber by using a syringe pump at a speed of $0.25 \mathrm{~mL} \mathrm{~min}^{-1}$. When a pollen particle passes through the laser light and causes significant light scatter that exceeds a certain threshold, the camera captures time-lapse images in the corresponding field of view at 15 images per second. The optical settings were: brightness 100 , contrast 40 , sharpness 6 , and gain 400. Based on image data and analysis conducted using the Visual Spreadsheet software (Fluid Imaging Technologies), particle characteristics such as number concentration, edge gradient, and circularity were computed.

For $\mathrm{pH}$-dependent studies, five different $\mathrm{pH}$ conditions were tested: $2,4,7,10$, and 14 , and the solutions were prepared by the addition of $2 \mathrm{M} \mathrm{HCl}$ or $2 \mathrm{M} \mathrm{KOH}$ to deionized water. For each $\mathrm{pH}$ condition, $3 \mu \mathrm{L}$ of pollen microgel sample was mixed thoroughly with $700 \mu \mathrm{L}$ of the corresponding $\mathrm{pH}$ solution. After an incubation period of $5 \mathrm{~min}$, the pollen particles were dispersed by pipetting and then $200 \mu \mathrm{L}$ of the pollen suspension was passed through the flow cell.

For ion-dependent studies, aqueous solutions of $\mathrm{KCl}, \mathrm{MgCl}_{2}, \mathrm{CaCl}_{2}, \mathrm{SrCl}_{2}$, or Fe $\left(\mathrm{NO}_{3}\right)_{3}$ in deionized water were used. The solutions were prepared at the following ion concentrations: 5, 10, 20,50, and $100 \mathrm{mM}$. For each condition, $200 \mu \mathrm{L}$ of pollen microgel was mixed thoroughly with $3 \mathrm{~mL}$ of the ion solution in a $10 \mathrm{~mL}$ conical centrifuge tube and incubated for $5 \mathrm{~min}$. For control, deionized water was used as the dispersion medium in the absence of added ions. After an incubation period of $5 \mathrm{~min}, 200 \mu \mathrm{L}$ of the pollen microgel suspension was passed through the flow cell. The rest of the mixture was centrifuged at $5000 \times g$ for $5 \mathrm{~min}$. The resulting supernatant was discarded and $600 \mu \mathrm{L}$ of an aqueous solution containing an equivalent molar concentration of EDTA was added. After vortexing and an incubation period of $5 \mathrm{~min}, 200 \mu \mathrm{L}$ of the EDTA solution was passed through the flow cell. All the experiments were conducted in triplicate and 500 particles were used for analysis.

Fourier-transform infrared (FTIR) spectroscopy. Before experiment, the pollen gel samples were frozen at $-20^{\circ} \mathrm{C}$ for $24 \mathrm{~h}$ and then lyophilized in a freeze dryer (Labconco, Kansas City, MO, USA) under 0.008 mbar vacuum pressure for 2 days. FTIR measurements were performed on the freeze-dried samples by using a PerkinElmer spectrometer (PerkinElmer, Waltham, MA, USA) with a diamond cell attenuated total reflection (ATR) accessory module. Reflectance infrared spectra were collected at $4000-650 \mathrm{~cm}^{-1}$, with 16 scans per measurement and 3 replicate measurements per sample. Background spectra were collected prior to sample readings and automatically subtracted from each measurement. A baseline correction procedure was carried out using the Spectrum 10 software (PerkinElmer). Following baseline correction, each spectrum was standardized as previously reported $^{34}$.

Scanning electron microscopy (SEM). For the defatted samples, the pollen particles were dried in a freeze dryer (Labconco) under 0.008 mbar vacuum pressure for two days. For the microgel samples, $3 \mu \mathrm{L}$ of the sample was dispersed in $200 \mu \mathrm{L}$ of the appropriate medium in a $1.5 \mathrm{~mL}$ microcentrifuge tube and then frozen with liquid nitrogen for $2 \mathrm{~min}$, followed by drying in a freeze dryer for two days. The dried samples were spread and immobilized on a sample holder with copper tape and sputter-coated with a $20-\mathrm{nm}$ thick gold film using a JFC-1600 Auto Fine Coater (JEOL, Tokyo, Japan; operating settings, $20 \mathrm{~mA}$ for $80 \mathrm{~s}$ ). For cross-sectional observation, the dried samples were adhered onto a piece of doublesided copper tape $(2 \mathrm{~cm} \times 1.3 \mathrm{~cm})$ and dipped into liquid nitrogen for $5 \mathrm{~min}$. Then, multiple cuts were conducted across the frozen sample with a surgical blade (B. Braun Melsungen AG, Melsungen, Germany). Finally, the pollen-adhered copper tape was dried in a freeze dryer for two days. Field-emission SEM imaging was performed using a JSM-7600F Schottky field-emission scanning electron microscope (JEOL) at an accelerating voltage of $5.00 \mathrm{kV}$ under various magnification levels (between $\times 1500$ and $\times 15000$ ).

EDC/NHS activation of pollen particles. The carboxyl acid functional groups on the surface of pollen particles were activated by treatment with1-ethyl-3-(3dimethylaminopropyl) carbodiimide hydrochloride (EDC, Sigma-Aldrich) and Nhydroxysuccinimide (NHS, Sigma-Aldrich). First, pollen samples $(20 \mathrm{mg})$ were

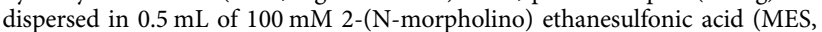
Sigma-Aldrich) buffer (0.5 M NaCl, pH 6.0). Then, EDC (5 mg) and NHS (15 mg) were added to the pollen suspension and quickly dispersed by vortexing. After an incubation period of $30 \mathrm{~min}$ on a rocking platform shaker, the suspension was centrifuged $(555 \times g, 5 \mathrm{~min})$ in order to remove the supernatant containing unreacted EDC and NHS. Then, $0.5 \mathrm{~mL}$ of PBS (pH 7.4) was added to re-suspend the pollen. Immediately after resuspension, the pollen samples were either used immediately or frozen by liquid nitrogen and placed in a freeze-dryer under 0.008 mbar vacuum pressure for 4-6h. Freeze-dried pollen samples were stored in a dry cabinet until further use.

Immobilization of pollen particles. Glass coverslips $(25 \mathrm{~mm} \times 75 \mathrm{~mm}$, ibidi $\mathrm{GmbH}$, Planegg, Germany) were sequentially rinsed with water and ethanol, dried with a stream of nitrogen gas, and treated with oxygen plasma for 1 min using an Expanded Plasma Cleaner (PDC-002, Harrick Plasma, Ithaca, NY, USA). After cleaning, the glass coverslips were immediately soaked in a $2 \%$ (vol/ $/ \mathrm{vol})$ solution of (3-Aminopropyl) triethoxysilane (APTES, Sigma-Aldrich) in $95 \%$ ethanol to functionalize the surface. After $30 \mathrm{~min}$, the coverslips were sequentially rinsed with deionized water and $95 \%$ ethanol for a total of 3 times before drying with a stream of nitrogen gas. As-prepared APTES-treated coverslips were kept in a dry cabinet and used within 2 weeks. Then, activated pollen particles were dispersed in PBS buffer and injected onto an APTES-treated glass coverslip that was enclosed within a microfluidic flow-through chamber (sticky-Slide VI 0.4, ibidi GmbH) at a flow rate of $300 \mu \mathrm{L} \mathrm{min}^{-1}$, as controlled by an Ismatec Reglo Digital peristaltic pump (Cole-Parmer GmbH, Wertheim, Germany). After checking that the density of attached pollen particles was within a sufficient range, the chamber was thoroughly washed by flowing PBS buffer at a flow rate of $3 \mathrm{~mL} \mathrm{~min}^{-1}$.

Confocal laser scanning microscopy (CLSM). Pollen gel samples in suspension $(\sim 20 \mu \mathrm{L})$ were pipetted onto a thin glass slide $(24 \mathrm{~mm} \times 75 \mathrm{~mm}$, CellPath Ltd, Newton, UK). CLSM imaging was performed using a Zeiss LSM710 microscope (Carl Zeiss, Oberkochen, Germany) equipped with three spectral reflection/fluorescence detection channels, six laser lines (405/458/488/514/561/633 nm), and connected to a Z1 inverted microscope (Carl Zeiss). A $\times 20$ optical lens was used for imaging and at least two images were obtained per sample. Imaging was performed under the laser excitation channel at $488 \mathrm{~nm}(12.0 \%)$. Fluorescence signals from the samples were collected in photomultiplier tubes equipped with emission filters at $495-550 \mathrm{~nm}$. Plane mode scanning was performed with a pixel dwell of $12.6 \mu \mathrm{s}$. Optimized imaging conditions were used for other types of pollen samples as follows: For immunolabeled-pollen particles, the samples were gently suspended by hand-shaking and then pipetted $(\sim 20 \mu \mathrm{L})$ onto a thin glass slide. Laser excitation channels were set at $488 \mathrm{~nm}(3.5 \%)$ and the emission filter was set at $493-634 \mathrm{~nm}$ Plane mode scanning was performed with a pixel dwell of $1.0 \mu$ s. For the immunolabeling studies, all imaging conditions were identical in order to compare the fluorescence intensity of each sample. For tethered pollen samples, imaging was performed with two excitation channels, such as $405 \mathrm{~nm}$ and $488 \mathrm{~nm}$, with two respective emission filters: $416-477 \mathrm{~nm}$ and $430-740 \mathrm{~nm}$. Plane mode scanning was performed with a pixel dwell of $1.0 \mu$ s. Image processing was performed with Fiji software (available at http://fiji.sc/ [Accessed February 21, 2019]).

Time-Lapse microscopy imaging. Microscopy experiments were conducted using an Eclipse Ti-E inverted microscope (Nikon, Tokyo, Japan) with a CFI Super Plan Fluor ELWD $\times 20$ or $\times 40(\mathrm{NA}=0.45$ or 0.60$)$ objective lens (Nikon), and images were collected with an iXon3 512 pixel $\times 512$ pixel EMCCD camera (Andor Technology, Belfast, UK). A halogen lamp, light power supply (TI-PS100W, Nikon) connected to TI-DH diascopic pillar illuminator (Nikon), was used to illuminate the pollen samples. As described above, the pollen particles were immobilized on an APTES-treated glass coverslip and the coated coverslip was then enclosed within a microfluidic flow-through chamber $(25.5 \mathrm{~mm} \times 75.5 \mathrm{~mm})$ with a $\sim 200 \mu \mathrm{L}$ volume channel. Liquid samples were introduced at a flow rate of $300 \mu \mathrm{L} \mathrm{min}^{-1}$, as controlled by a peristaltic pump (Cole-Parmer GmbH). During the swelling/de-swelling experiments, the bulk solution in the measurement chamber was exchanged and time-lapse images were captured in 0.4-s time intervals. Data processing was performed in MATLAB (MathWorks, Natick, MA, USA), ImageJ (National Institutes of Health, Bethesda, MD, USA) and OriginPro 8.5 (OriginLab, Northampton, MA, USA) software programs (Supplementary Fig. 29).

Immunolabeling studies. In order to detect the presence and esterification state of pectin molecules within the intine layer, JIM 5 and JIM 7 antibodies were used according to previously defined protocols ${ }^{17,35}$. Defatted pollen grain $(10 \mathrm{mg})$ or asprepared pollen microgel $(10 \mu \mathrm{L})$ samples were washed with phosphate-buffered saline (PBS, $1 \mathrm{~mL}$ ) and then incubated in $2 \%$ bovine serum albumin (BSA) in PBS $(1 \mathrm{~mL})$ for $30 \mathrm{~min}$. The pollen particles were then incubated overnight in a shaker (Gaia Science Pte Ltd, Singapore) with a rotation speed of $200 \mathrm{rpm}$ at $4^{\circ} \mathrm{C}$ in the presence of a primary antibody (JIM 5 or JIM 7) (PlantProbes, Leeds, UK, Cat. No. JIM5 and Cat. No. JIM7) at 1:20 dilutions in PBS containing 0.2\% BSA. After washing twice with fresh PBS, the samples were incubated with Alexa Fluor 488 AffiniPure Goat Anti-Rat IgG $(\mathrm{H}+\mathrm{L})$ secondary antibody (Jackson ImmunoResearch Laboratories, West Grove, PA, USA, Cat. No. 112-545-003), diluted 1:100 in PBS containing $0.2 \% \mathrm{BSA}$, for $1 \mathrm{~h}$ in a dark environment. Antibody-labeled pollen particles were examined immediately with a confocal laser scanning microscope (Zeiss LSM 710) and without any antifade reagents. Pollen grains without primary and/or secondary antibodies were used as controls. 
Force-distance measurements for mechanical characterization. The mechanical properties of defatted pollen and microgel particles were characterized by conducting AFM force-distance (or load versus displacement) measurements. This depth-sensing AFM indentation approach enables the quantitative determination of the Young's modulus of the shell material ${ }^{36,37}$. For wet samples, pollen microgel samples $(5 \mu \mathrm{L})$ were dispersed in the appropriate medium $(50 \mu \mathrm{L})$, and the mixture was spread onto a petri dish (Nunc, Roskilde, Denmark) by pipette. Then, excess liquid was aspirated and the partially hydrated samples remained on the glass slide for experimental characterization. For dry specimens, the pollen sample was directly spread onto the surface of a petri dish before measurement. Intact pollen particles were chosen for extracting the Young's modulus of the exine layer and broken pollen particles, which exposed the inner layer, were chosen to determine the Young's modulus of the intine layer. In order to avoid the substrate effect, we followed the $10 \%$ depth rule where the indentation depth should be less than $1 / 10$ th of the layer thickness ${ }^{38}$. The average thickness of the exine layer was $\sim 0.6$ $\mu \mathrm{m}$ for defatted pollen particles and $0 \mathrm{~h} \mathrm{KOH}$-treated pollen specimens, and became thinner with increasing $\mathrm{KOH}$ treatment time $(\sim 0.5 \mu \mathrm{m})$. Thus, the indentation depths for exine measurements were set in the range of $20-60 \mathrm{~nm}$ under indentation loads of 3-6 $\mu \mathrm{N}$. On the other hand, the intine layer was swollen in wet conditions, with a thickness range from 0.5 to $1.5 \mu \mathrm{m}$. Thus, for dry samples, the indentation depths of intine measurements were carefully limited up to $\sim 60$ $\mathrm{nm}$, while for wet samples, the indentation depths were in the range of 50 to 100 $\mathrm{nm}$. For all measurements, the NX-10 AFM instrument (Park Systems, Suwon, South Korea) was used with two AFM probes: (i) an aluminum reflex-coated silicon cantilever PPP-NCHR (Nanosensors, Neuchâtel, Switzerland) with a typical spring constant of $\sim 42 \mathrm{~N} \mathrm{~m}^{-1}$ and a tip end radius of $5 \mathrm{~nm}$; and (ii) a diamond cantilever TD26135 (Micro Star Technology, Huntsville, TX) with a spring constant of $\sim 150 \mathrm{~N} \mathrm{~m}^{-1}$ and a tip end radius of $5 \mathrm{~nm}$. The tips were shaped as polygon-based pyramid with a half-cone angle of $20^{\circ}$. We confirmed that both AFM probes provided almost identical values of Young's moduli for exine and intine layers regardless of the various indenting parameters such as maximum contact forces, contact time or approach speed (Supplementary Fig. 30). Thus, measurements were conducted at various positions (more than 16 data points) in a $5 \mu \mathrm{m} \times 5 \mu \mathrm{m}$ area at an approach speed of $0.8 \mu \mathrm{m} \mathrm{s}^{-1}$ with a maximum loading force of $4.8 \mu \mathrm{N}$ and zero contact time. Before experiments, the AFM cantilever was rinsed with water and ethanol, and treated with a UV light cleaner for $30 \mathrm{~min}$ in order to remove organic contaminants. The spring constant and sensitivity of the deflection signal were also calibrated by recourse to the thermal vibration of the AFM cantilever ${ }^{39}$ by employing the commercial software (XEP, Park Systems). The force-versus-displacement curves were corrected by subtracting the deflection distance of the AFM probe from the total displacement.

For data analysis, we assumed that both the exine and intine layers were isotropic. Also, we used an AFM probe with a tip end radius of $5 \mathrm{~nm}$, and thus the indentation depths $(20-100 \mathrm{~nm})$ were significantly larger than the tip end radius. Thus, the classical Hertz model was used for data analysis ${ }^{37,40}$. The Hertz model ${ }^{36}$ was fitted to the force-versus-displacement curves using a commercial software analysis program (XEI, Park Systems) and the Python script language

(Supplementary Fig. 30). We treated the geometry of the AFM tip as a parabolic model whereby it has a tip radius of $R_{\mathcal{c}}$, so that the force $(F)$ can be expressed as:

$$
F=\frac{4 \sqrt{ } R_{c}}{3} \frac{E}{1-v^{2}} \delta^{3 / 2},
$$

where $E$ is Young's modulus, $v$ is the Poisson's ratio, and $\delta$ is the indentation depth. The Poisson's ratio $v$ was set at 0.5 , which is typical for natural materials.

Numerical modeling for swelling/deswelling of pollen microgel particles. For pollen grains, the intine layer is naturally hydrophobic due to highly esterified pectin, whereas the exine layer consists of sporopollenin that contains lipids and pollenkitt (pollen cement). Thus, the pollen shell is inherently hydrophobic and not swellable in its natural form. After the chemical treatment, the highly esterified pectin becomes de-esterified and hydrophilic while the exine layer becomes porous (due to removal of pollen cement). In addition, due to chemical treatment, the cytoplasm is also fully removed and thus the internal cavity is fully empty (Supplementary Fig. 2). Also, since the cytoplasmic contents, including cells and cellular debris, have to be released through the three apertures, the once-continuous intine layer is also ruptured. Thus, in the modeling, the two-layer pollen shell was fully discretized through the entire layer thickness at the apertures as shown in Supplementary Fig. 2. As a result, a pollen microgel particle has a hollow shell structure with three apertures, consisting of the outer exine and inner intine layers. Thus, the volumetric increase in a pollen microgel particle is caused by the interplay of the swollen intine layer and relatively stiffer exine layer. In particular, we carefully defined the swelling/deswelling behavior of the hydrogel-like intine layer due to water absorption and desorption depending on the osmotic pressure and inflation/ deflation of the hyperelastic exine due to swelling-induced mechanical pressure of the underlying intine layer. Moreover, the three apertures are open, which allows rapid water intake into the internal cavity of the hollow particle that is concomitant with inflation of the pollen exine. Taken together, two key factors affect the swelling/deswelling behavior of the pollen microgel particle system: (1) intine uptakes ionic solution from the surrounding medium to generate a considerable osmotic pressure, which is the driving force for inflating the outer exine; and (2) the stiffer exine along with the three apertures exerts an inhomogeneous constraint on intine swelling. Therefore, the swelling-induced mechanical pressure exerted at the outer surface of the intine layer $\left(P_{i, \text { swelling }}\right)$ due to osmotic pressure decreases as swelling proceeds. The intine swelling pressure $\left(P_{i, \text { swelling }}\right)$ equals the equilibrium pressure that is required to inflate the exine layer $\left(P_{e, \text { inflation }}\right)$. The swelling ratio of the intine, $\lambda_{i}$, is equal to the inflation ratio of exine, $\lambda_{e}$ since the intine layer is tightly bound to the exine layer (Supplementary Fig. 13a). Details of the overall approach behind the multiphysics model for intine layer and finite element analysis $(\mathrm{FEA})^{27-29}$ for exine layer are described in Supplementary Methods.

Statistical analysis. Statistical analysis was performed using the Origin 2018 software package (OriginLab). Unpaired Student's $t$-test and one-way ANOVA with Tukey's multiple comparison tests were used to calculate the statistical significance of data sets. A $P$ value of less than 0.05 was considered statistically significant. Data are presented as the mean \pm standard deviation, wherever appropriate.

\section{Data availability}

All the data supporting the findings of this study are available from the corresponding authors upon reasonable request. The source data underlying Figs. 1d, 2c, f, and 3a, b, Supplementary Figs. 4, 7, 8a, b, 9b, c, 11, 12, 19-28, and 30a-e, and Supplementary Table 1 are provided as a Source Data file.

\section{Code availability}

The computer code and algorithm that support the findings of this study are available from the corresponding author upon reasonable request.

Received: 16 April 2019; Accepted: 23 February 2020;

Published online: 19 March 2020

\section{References}

1. Faegri, K. \& Van der Pijl, L. Principles of Pollination Ecology (Elsevier, 2013).

2. Tylianakis, J. M. The global plight of pollinators. Science 339, 1532-1533 (2013).

3. Birks, H. J. B., Birks, H. H. \& Ammann, B. The fourth dimension of vegetation. Science 354, 412-413 (2016).

4. de Miranda Chaves, S. A. Pollen Grains, Landscapes, and Paleoenvironments. 205-222 (SciELO, 2014).

5. Blackmore, S., Wortley, A. H., Skvarla, J. J. \& Rowley, J. R. Pollen wall development in flowering plants. N. Phytol. 174, 483-498 (2007).

6. Radja, A., Horsley, E. M., Lavrentovich, M. O. \& Sweeney, A. M. Pollen cell wall patterns form from modulated phases. Cell 176, 856-868 (2019).

7. Mackenzie, G., Boa, A. N., Diego-Taboada, A., Atkin, S. L. \& Sathyapalan, T Sporopollenin, the least known yet toughest natural biopolymer. Front. Mater. 2, 66 (2015).

8. Katifori, E., Alben, S., Cerda, E., Nelson, D. R. \& Dumais, J. Foldable structures and the natural design of pollen grains. Proc. Natl Acad. Sci. USA 107, 7635-7639 (2010)

9. Couturier, E., Dumais, J., Cerda, E. \& Katifori, E. Folding of an opened spherical shell. Soft Matter 9, 8359-8367 (2013).

10. Heslop-Harrison, J. Pollen germination and pollen-tube growth. Int. Rev. Cytol. 107, 1-78 (1980).

11. Bosch, M. \& Hepler, P. K. Pectin methylesterases and pectin dynamics in pollen tubes. Plant Cell 17, 3219-3226 (2005).

12. Erdtman, G. Pollen morphology and plant taxonomy: angiosperms. Vol. 1 (Brill Archive, 1986).

13. Rojas, E. R., Hotton, S. \& Dumais, J. Chemically mediated mechanical expansion of the pollen tube cell wall. Biophys. J. 101, 1844-1853 (2011).

14. Heslop-Harrison, Y. \& Heslop-Harrison, J. The microfibrillar component of the pollen intine some structural features. Ann. Bot. 50, 831-842 (1982).

15. Bosch, M., Cheung, A. Y. \& Hepler, P. Pectin methylesterase, a regulator of pollen tube growth. Plant Physiol. 138, 1334-1346 (2005).

16. Wolf, S., Hématy, K. \& Höfte, H. Growth control and cell wall signaling in plants. Annu. Rev. Plant Biol. 63, 381-407 (2012).

17. Vieira, A. M. \& Feijó, J. A. Hydrogel control of water uptake by pectins during in vitro pollen hydration of Eucalyptus globulus. Am. J. Bot. 103, 437-451 (2016).

18. Knox, J. P., Linstead, P. J., King, J., Cooper, C. \& Roberts, K. Pectin esterification is spatially regulated both within cell walls and between developing tissues of root apices. Planta 181, 512-521 (1990).

19. Parre, E. \& Geitmann, A. Pectin and the role of the physical properties of the cell wall in pollen tube growth of Solanum chacoense. Planta 220, 582-592 (2005). 
20. Saunders, B. R. et al. Microgels: from responsive polymer colloids to biomaterials. Adv. Colloid Interface Sci. 147, 251-262 (2009).

21. Gibbs, F. W. The history of the manufacture of soap. Ann. Sci. 4, 169-190 (1939).

22. Kiser, P. F., Wilson, G. \& Needham, D. A synthetic mimic of the secretory granule for drug delivery. Nature 394, 459 (1998).

23. Zhao, B. \& Moore, J. S. Fast $\mathrm{pH}$-and ionic strength-responsive hydrogels in microchannels. Langmuir 17, 4758-4763 (2001).

24. BeMiller, J. N. An Introduction to Pectins: Structure and Properties. Vol. 310 (ACS Symposium Series, 1986).

25. Michard, E., Simon, A. A., Tavares, B., Wudick, M. M. \& Feijó, J. A. Signaling with ions: the keystone for apical cell growth and morphogenesis in pollen tubes. Plant Physiol. 173, 91-111 (2017).

26. Pacinia, E. \& Hesseb, M. Pollenkitt-Its composition, forms and functions. Flora 200, 399-415 (2005).

27. Hong, W., Zhao, X. \& Suo, Z. Large deformation and electrochemistry of polyelectrolyte gels. J. Mech. Phys. Solids 58, 558-577 (2010).

28. Hong, W., Zhao, X., Zhou, J. \& Suo, Z. A theory of coupled diffusion and large deformation in polymeric gels. J. Mech. Phys. Solids 56, 1779-1793 (2008).

29. Liu, X. et al. Ingestible hydrogel device. Nat. Commun. 10, 1-10 (2019).

30. Matamoro-Vidal, A. et al. Links between morphology and function of the pollen wall: an experimental approach. Bot. J. Linn. Soc. 180, 478-490 (2016).

31. Mundargi, R. C. et al. Natural sunflower pollen as a drug delivery vehicle. Small 12, 1167-1173 (2016).

32. Potroz, M. G. et al. Plant-Based hollow microcapsules for oral delivery applications: toward optimized loading and controlled release. Adv. Funct. Mater. 27, 1700270 (2017).

33. Mundargi, R. C. et al. Extraction of sporopollenin exine capsules from sunflower pollen grains. RSC Adv. 6, 16533-16539 (2016).

34. Jardine, P. E., Abernethy, F. A., Lomax, B. H., Gosling, W. D. \& Fraser, W. T. Shedding light on sporopollenin chemistry, with reference to UV reconstructions. Rev. Palaeobot. Palynol. 238, 1-6 (2017).

35. Clausen, M. H., Willats, W. G. \& Knox, J. P. Synthetic methyl hexagalacturonate hapten inhibitors of anti-homogalacturonan monoclonal antibodies LM7, JIM5 and JIM7. Carbohydr. Res. 338, 1797-1800 (2003).

36. Parre, E. \& Geitmann, A. More than a leak sealant. The mechanical properties of callose in pollen tubes. Plant Physiol. 137, 274-286 (2005).

37. Qu, Z. \& Meredith, J. C. The atypically high modulus of pollen exine. J. R. Soc. Interface 15, 20180533 (2018).

38. Zeng, Z. \& Tan, J.-C. AFM nanoindentation to quantify mechanical properties of nano-and micron-sized crystals of a metal-organic framework material. ACS Appl. Mater. Interfaces 9, 39839-39854 (2017).

39. Hutter, J. L. \& Bechhoefer, J. Calibration of atomic-force microscope tips. Rev. Sci. Instrum. 64, 1868-1873 (1993).

40. Neumann, T. Determining the elastic modulus of biological samples using atomic force microscopy. JPK Instrum. Appl. Rep. 1-9 (2008).

\section{Acknowledgements}

This work was supported by the National Research Foundation of Singapore through a Competitive Research Programme grant (NRF-CRP10-2012-07) and by the Ministry of Education of Singapore through a AcRF Tier 1 grant 2017-T1-001-246 (RG51/17).

\section{Author contributions}

J.S., S.S., and N.-J.C. designed the study. T.-F.F., S.P., Y.S., H.C., M.S.I., N.M., and Y.Y. carried out experiments and collected data. Q.S., X.Z., Q.L., and H.L. contributed to numerical modeling and theoretical analysis. N.-J.C. initiated the idea. All authors contributed to data analysis and writing the paper.

\section{Competing interests}

N.-J.C. is a co-inventor on patent no. WO2019147190A1. The remaining authors declare no competing interests.

\section{Additional information}

Supplementary information is available for this paper at https://doi.org/10.1038/s41467020-15294-w.

Correspondence and requests for materials should be addressed to H.L., J.S., S.S. or N.-J.C.

Peer review information Nature Communications thanks Idris Sargın, Wei Hong and the other, anonymous, reviewer(s) for their contribution to the peer review of this work. Peer reviewer reports are available.

Reprints and permission information is available at http://www.nature.com/reprints

Publisher's note Springer Nature remains neutral with regard to jurisdictional claims in published maps and institutional affiliations.

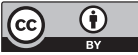

Open Access This article is licensed under a Creative Commons Attribution 4.0 International License, which permits use, sharing, adaptation, distribution and reproduction in any medium or format, as long as you give appropriate credit to the original author(s) and the source, provide a link to the Creative Commons license, and indicate if changes were made. The images or other third party material in this article are included in the article's Creative Commons license, unless indicated otherwise in a credit line to the material. If material is not included in the article's Creative Commons license and your intended use is not permitted by statutory regulation or exceeds the permitted use, you will need to obtain permission directly from the copyright holder. To view a copy of this license, visit http://creativecommons.org/ licenses/by/4.0/

(C) The Author(s) 2020 